\title{
Connecting orbits in perturbed systems
}

\author{
Fritz Colonius • Thorsten Hüls • \\ Martin Rasmussen
}

\begin{abstract}
We apply Newton's method and continuation techniques to determine heteroclinic connections in perturbed non-autonomous differential equations which do not exist for the underlying unperturbed system. This approach is particularly useful in a higherdimensional context, where the numerical computation of invariant manifolds is very expensive. A detailed discussion of a four-dimensional model is presented, which describes a pendulum coupled to a harmonic oscillator.
\end{abstract}

Keywords Newton method · Connecting orbits . Perturbations · Control

Supported by CRC 701 'Spectral Structures and Topological Methods in Mathematics'.

Research supported by a Marie Curie Intra European Fellowship of the European Community (Grant Agree Number: 220638)

F. Colonius

Institut für Mathematik, Universität Augsburg,

86135 Augsburg, Germany

T. Hüls

Fakultät für Mathematik, Universität Bielefeld, 33501 Bielefeld, Germany

M. Rasmussen ( $\varangle$ )

Department of Mathematics, Imperial College, London SW72AZ, UK

e-mail:m.rasmussen@imperial.ac.uk

\section{Introduction}

The determination of connecting orbits in dynamical systems has found widespread interest in numerical analysis and various engineering disciplines. It is the purpose of the present note to show how a Newton method can be applied for the computation of heteroclinic orbits for perturbed non-autonomous ordinary differential equations.

We consider families of ordinary differential equations of the form

$\dot{x}(t)=f_{0}(x(t))+\sum_{i=1}^{m} u_{i}(t) f_{i}(x(t)), \quad u \in \mathcal{U}$,

where $f_{i}: \mathbb{R}^{d} \rightarrow \mathbb{R}^{d}, i=0,1, \ldots, m$, are smooth vector fields and the functions $u \in \mathcal{U}$ are defined on $\mathbb{R}$ with values in a fixed set $U \subset \mathbb{R}^{m}$ with $0 \in U$. The solutions corresponding to initial conditions $x(0)=\xi$ and functions $u \in \mathcal{U}$ are denoted by $\varphi(\cdot, \xi, u)$, and we assume that the solutions exist for all $t \in \mathbb{R}$.

We are interested in finding orbits of the perturbed system (1) which connect points near equilibria $\xi_{-}$and $\xi_{+}$of the system with $u_{i} \equiv 0$ for $i=0,1, \ldots, m$. We assume here that such an orbit does not exist for the unperturbed differential equation $\dot{x}=f_{0}(x)$, and via continuation techniques, we also analyze the domain of perturbations which admit such an orbit.

Problems of this type occur in various contexts: In control theory, for instance, the functions $u$ are interpreted as control functions which can be chosen in or- 
der to steer the system from $\xi_{-}$to $\xi_{+}$. If one interprets $u$ as realizations of random processes, the existence of a connecting orbit implies that the system moves with positive probability from a neighborhood of $\xi_{-}$ to a neighborhood of $\xi_{+}$. Often, this precedes loss of stability for a stable equilibrium between $\xi_{-}$and $\xi_{+}$, which was observed, e.g., in Colonius, Kreuzer, Marquardt and Sichermann [8].

If system (1) is a perturbation from a Hamiltonian system, the existence of connecting orbits can be determined by analyzing the zeros of Melnikov functions (see Melnikov [21], Guckenheimer and Holmes [14], and in a stochastic context, we refer to Wiggins [25], Frey and Simiu [11], Simiu [23] and Zhu and Liu [26]). This theory has applications to many mechanical systems, in particular, roll motion in the analysis of ship stability has been analyzed using these arguments.

In [8], connecting orbits for control systems in dimension $d=2$ have been determined by either computing reachable sets in positive and negative time and then taking their intersection, or alternatively, by computing stable and unstable manifolds and then taking their intersection. Both methods are computationally expensive, and it appears difficult to apply them in higher dimensions.

On the other hand, Newton methods for computing homoclinic and heteroclinic orbits of maps have been developed in Beyn and Kleinkauf [4], Beyn, Hüls, Kleinkauf and Zou [3] and Beyn and Hüls [2], with a view toward non-hyperbolic equilibria. These numerical methods have been applied to a number of examples given by maps. It is the purpose of the present paper to show that these methods can also be applied to control problems and to show that they can successfully be used in higher-dimensional problems arising from time- $T$ maps of ordinary differential equations. We illustrate this in a four-dimensional system that has been analyzed in Zhu and Liu [26] via Melnikov's method.

We remark that the problem to connect two points in the state space by a finite time trajectory is a standard controllability problem in control theory. Analytically, it may be treated by applying fixed-point theorems, and numerically, it can by embedded into an optimal control problem: if two points can be connected by a controlled trajectory, they can (under mild assumptions) also be connected by a time or energy optimal trajectory. The resulting optimal control problem leads to two-point boundary value problems, which then are solved numerically. In the context of computer assisted proofs and interval arithmetics (see, e.g., Jaulin, Kiefer, Didrit and Walter [18]), where errors of floating point numerics are taken into account, the paper Colonius and Kapela [5] approached this problem by computing switching times for piecewise constant controls. This can be reformulated as the problem to find a zero of a function and an interval Newton method is applied. Applications in [5] of this (numerically very expensive) method are also restricted to two-dimensional systems where delicate controllability problems occur: a Takens-Bogdanov system from Häckl and Schneider [15] concerning the existence of control-homoclinic orbits and the escape equation from Gayer [12] related to bifurcation problems for control sets. We remark that the results in the present paper might also be used in order to find starting values for the methods from [5]; here good initial guesses are of primary importance.

This paper is organized as follows. In the ensuing section, we briefly recapitulate the method from [2] for computing connecting orbits. In Sect. 3, we demonstrate this method for the study of a differential equation which models ship roll motion. Section 4 presents our results on a four-dimensional system, which describes a pendulum coupled to a harmonic oscillator under deterministic or random perturbations and, finally, Sect. 5 draws some conclusions.

\section{A Newton method for computing connecting orbits}

In this section, we adapt an algorithm for the approximation of connecting orbits, which was introduced in Hüls [16], to our situation of perturbed nonautonomous ordinary differential equations. More precisely, we are not dealing directly with the differential equation (1) but with a suitable discretization, given by a time- $T$ map, resulting in a discrete system of the form

$x^{n+1}=f\left(x^{n}, u^{n}\right), \quad n \in \mathbb{Z}$,

where $f: \mathbb{R}^{d} \times \mathbb{R} \rightarrow \mathbb{R}^{d}$ is a sufficiently smooth diffeomorphism and $\left(u^{n}\right)_{n \in \mathbb{Z}}$ is a sequence of control parameters. We choose constant control on $\mathbb{Z}^{-}$and 
Fig. 1 Sketch of a heteroclinic orbit of (2)

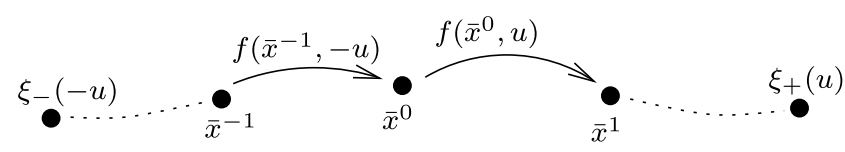

on $\mathbb{Z}_{0}^{+}$, i.e.,

$u^{n}= \begin{cases}-u & \text { for } n \in \mathbb{Z}^{-}, \\ u & \text { for } n \in \mathbb{Z}_{0}^{+} .\end{cases}$

Let $\xi_{-}(-u)$ and $\xi_{+}(u)$ be fixed points of $f(\cdot,-u)$ and $f(\cdot, u)$, respectively, and assume that there exists a heteroclinic orbit $\bar{x}_{\mathbb{Z}}=\left(\bar{x}^{n}\right)_{n \in \mathbb{Z}}$ which connects these fixed points, i.e.,

$\bar{x}^{n+1}=f\left(\bar{x}^{n}, u^{n}\right) \quad$ for $n \in \mathbb{Z} \quad$ and

$\lim _{n \rightarrow \pm \infty} \bar{x}^{n}=\xi_{ \pm}( \pm u)$,

\section{cf. Fig. 1.}

We obtain a finite approximation of the heteroclinic orbit $\bar{x}_{\mathbb{Z}}$ on the interval $J=\left[n_{-}, n_{+}\right] \cap \mathbb{Z}$ by computing a zero of the operator $\Gamma_{J}:\left(\mathbb{R}^{d}\right)^{J} \rightarrow\left(\mathbb{R}^{d}\right)^{J}$, where

$\Gamma_{J}\left(x_{J}\right)=\left(\begin{array}{c}x^{n+1}-f\left(x^{n}, u^{n}\right), n=n_{-}, \ldots, n_{+}-1 \\ b\left(x^{n_{-}}, x^{n_{+}}\right)\end{array}\right)$.

Here $b: \mathbb{R}^{d} \times \mathbb{R}^{d} \rightarrow \mathbb{R}^{d}$ is the so-called projection boundary operator, defined as

$b\left(x^{n_{-}}, x^{n_{+}}\right)=\left(\begin{array}{l}Y_{s}^{T} x^{n_{-}} \\ Y_{u}^{T} x^{n_{+}}\end{array}\right)$,

where $Y_{s}$ is a basis of the stable subspace of $D_{x} f\left(\xi_{-}(-u),-u\right)^{T}$ and $Y_{u}$ forms a basis of the unstable subspace of $D_{x} f\left(\xi_{+}(u), u\right)^{T}$. We compute $Y_{s}$ and $Y_{u}$ using an eigenvalue solver and normalize these vectors if appropriate; cf. Beyn [1]. It was not necessary to apply more sophisticated methods, such as Demmel, Dieci and Friedman [9].

For finding a zero of the non-linear operator $\Gamma_{J}$, we apply Newton's method, i.e., we choose a good initial guess $\left(x_{J}\right)_{0}$, for example,

$\left(x_{J}\right)_{0}=\left(\xi_{-}(-u), \ldots, \xi_{-}(-u), r, \xi_{+}(u), \ldots, \xi_{+}(u)\right)^{T}$

with a suitable $r$, calculate in the $n$-th step $\left(x_{J}\right)_{n+1}$ via

$$
\begin{aligned}
& D_{x} \Gamma_{J}\left(\left(x_{J}\right)_{n}, u_{J}\right)\left(\left(x_{J}\right)_{n+1}-\left(x_{J}\right)_{n}\right) \\
& \quad=-\Gamma_{J}\left(\left(x_{J}\right)_{n}, u_{J}\right)
\end{aligned}
$$

and stop this iteration if $\left\|\left(x_{J}\right)_{n+1}-\left(x_{J}\right)_{n}\right\|_{\infty}$ is less than a given tolerance. Note that $D_{x} \Gamma_{J}$ is a sparse matrix, and thus, one can solve the linear system (6) efficiently.

If both fixed points are hyperbolic, and the corresponding fiber bundles intersect transversally, it turns out that $\Gamma_{J}\left(x_{J}\right)=0$ has, for sufficiently large intervals $J$, a unique bounded solution $x_{J}$ in some $\delta$ neighborhood of the restricted exact solution $\bar{x}_{\mid J}$. The approximation error can be estimated as

$$
\begin{aligned}
\left\|x_{J}-\bar{x}_{\mid J}\right\|_{\infty} \leq & C\left\|b\left(\bar{x}^{n_{-}}, \bar{x}^{n_{+}}\right)\right\| \\
\leq & C\left(\left\|\bar{x}^{n_{-}}-\xi_{-}(-u)\right\|^{2}\right. \\
& \left.+\left\|\bar{x}^{n_{+}}-\xi_{+}(u)\right\|^{2}\right),
\end{aligned}
$$

cf. Hüls [16] and [17].

In a second step, we continue this orbit with respect to the control $u$ and determine in this way the range of controls for which heteroclinic orbits exist. For this task, the algorithm of pseudo arclength continuation is applied (see Keller [19] and Govaerts [13]) that is based on a Newton corrector scheme.

\section{A model for ship roll motion}

We discuss the differential equation

$$
\begin{aligned}
\dot{x}_{1}= & x_{2}, \\
\dot{x}_{2}= & -\beta_{1} x_{2}-\beta_{3} x_{2}^{3}-x_{1} \\
& +\alpha x_{1}^{3}+F \cos (\omega t)+u(t),
\end{aligned}
$$

which is used as a model for ship roll motion. This system was first studied in Kreuzer and Sichermann [20] without control $(u \equiv 0)$. We use their parameter values

$\alpha=0.674, \quad \beta_{1}=0.0231, \quad \beta_{3}=0.0375$

(see Colonius, Kreuzer, Marquardt and Sichermann [8], and we refer also to Wichtrey [24] for the periodically forced model). Without damping ( $\beta_{1}=$ $\beta_{3}=0$ ), the system is Hamiltonian, and the potential

$V(x):=\frac{x^{2}}{2}-\alpha \frac{x^{4}}{4}$ 


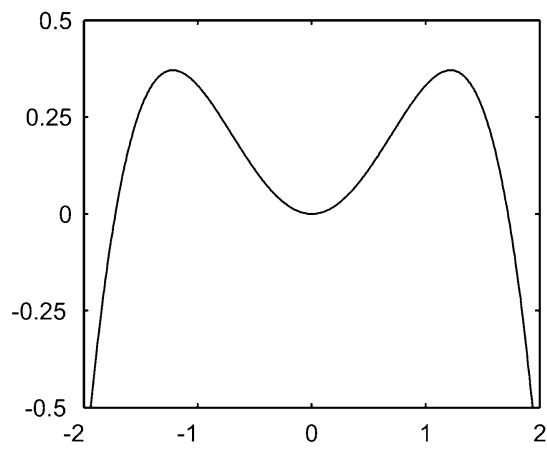

Fig. 2 The $M$-shaped potential

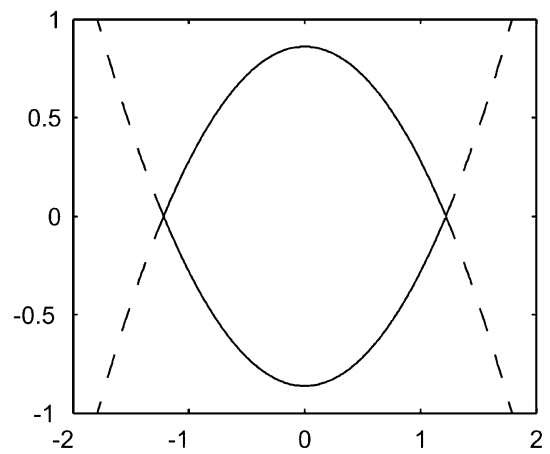

Fig. 3 The heteroclinic orbits of the associated Hamiltonian system on the right

is $M$-shaped (see Fig. 2).

In this case, the system has three equilibria, given by the asymptotically stable origin $\xi_{1}$ and two hyperbolic equilibria on the negative and positive $x_{1}$-axis, given by $\xi_{2}$ and $\xi_{3}$, respectively. The hyperbolic equilibria are connected by two heteroclinic orbits (see Fig. 3).

It is clear that in case of damping $\left(\beta_{1}, \beta_{3} \neq 0\right)$, these heteroclinic orbits do not persist. In the following, we will assume throughout that we have a periodic forcing of the system $(F \neq 0)$. As discussed in Wichtrey [24, Korollar 5.3], in case of damping, there exists a $F_{C}>0$ such that for all $F \geq F_{C}$, there is a heteroclinic connection from $\tilde{\xi}_{2}$ to $\tilde{\xi}_{3}$, where $\tilde{\xi}_{2}$ and $\tilde{\xi}_{3}$ are the fixed points of the time- $\frac{2 \pi}{\omega}$ map, which correspond to $\xi_{2}$ and $\xi_{3}$, respectively. Basically, this means that the unstable manifold of $\tilde{\xi}_{2}$ intersects with the stable manifold $\tilde{\xi}_{3}$ and vice versa. The main tool to prove this is Melnikov's method, and in [24], it is also shown that small forcing does not lead to a heteroclinic connection. We now want to demonstrate Newton's method from Sect. 2 to show that, in this case, a suitably chosen control function $u$ yields connecting orbits between $\tilde{\xi}_{2}$ and $\tilde{\xi}_{3}$.

In addition to (8), we choose

$\omega=\frac{5}{2} \quad$ and $\quad F=0.55$.

For these parameters the uncontrolled system has no heteroclinic connection [24]. We then applied Newton's method from Sect. 2 successfully for a control function

$u(t):= \begin{cases}-0.02 & \text { for } t \leq 0 \\ 0.02 & \text { for } t>0\end{cases}$

The corresponding fixed points of the time- $\frac{2 \pi}{\omega}$ map of (7) are given by

$\tilde{\xi}_{2}=\left(\begin{array}{c}-1.2914 \\ 0.0012\end{array}\right) \quad$ and $\quad \tilde{\xi}_{3}=\left(\begin{array}{l}1.1587 \\ 0.0012\end{array}\right)$,

and a finite approximation of length 10 of the corresponding orbit is given by

$$
\begin{aligned}
& \left(\begin{array}{l}
1.1587 \\
0.0012
\end{array}\right) \stackrel{u \equiv-0.02}{\longmapsto}\left(\begin{array}{l}
1.1587 \\
0.0012
\end{array}\right) \stackrel{u \equiv-0.02}{\longmapsto}\left(\begin{array}{l}
1.1585 \\
0.0009
\end{array}\right) \\
& \stackrel{u \equiv-0.02}{\longmapsto}\left(\begin{array}{c}
1.1509 \\
-0.0093
\end{array}\right) \stackrel{u \equiv-0.02}{\longmapsto}\left(\begin{array}{c}
0.9127 \\
-0.2963
\end{array}\right) \\
& \stackrel{u \equiv-0.02}{\longmapsto}\left(\begin{array}{l}
-0.8254 \\
-0.5719
\end{array}\right) \stackrel{u \equiv 0.02}{\longmapsto}\left(\begin{array}{l}
-1.2761 \\
-0.0218
\end{array}\right) \\
& \stackrel{u \equiv 0.02}{\longmapsto}\left(\begin{array}{c}
-1.2910 \\
0.0005
\end{array}\right) \stackrel{u \equiv 0.02}{\longmapsto}\left(\begin{array}{c}
-1.2914 \\
0.0012
\end{array}\right) \\
& \stackrel{u \equiv 0.02}{\longmapsto}\left(\begin{array}{c}
-1.2914 \\
0.0012
\end{array}\right)
\end{aligned}
$$

(see also Fig. 4).

It is also interesting to know if the heteroclinic orbit persists if we make changes in the control function. In the next section, we will use continuation methods for a four-dimensional system in order to follow the path of a heteroclinic orbit for different control functions $u$. 


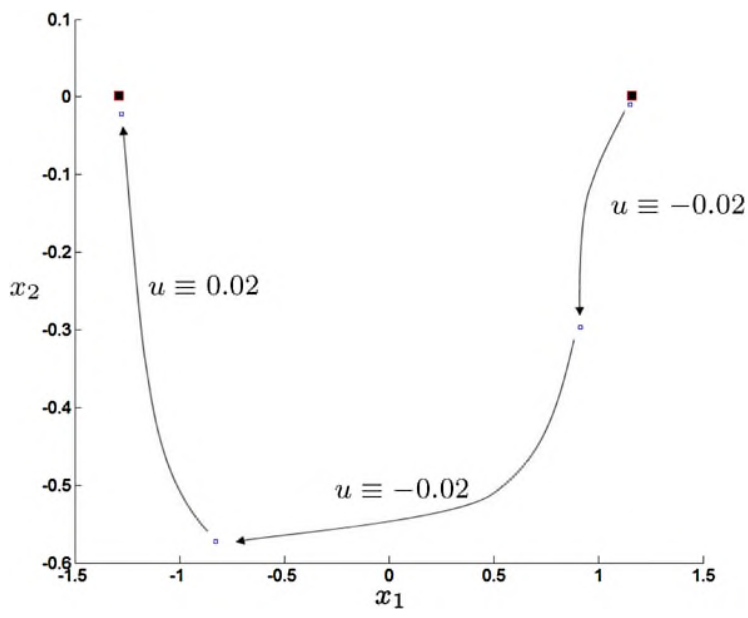

Fig. 4 Finite approximation of the heteroclinic orbit

\section{A pendulum coupled with a harmonic oscillator under perturbations}

We consider the following system from Zhu and Liu [26]:

$\dot{x}_{1}=x_{2}$,

$\dot{x}_{2}=-\sin x_{1}+\varepsilon\left(x_{3}-x_{1}\right)-\varepsilon \beta x_{2}+\varepsilon u(t)$,

$\dot{x}_{3}=x_{4}$,

$\dot{x}_{4}=-\omega^{2} x_{3}+\varepsilon\left(x_{1}-x_{3}\right)-\varepsilon \gamma x_{4}$,

where we have chosen the parameters

$\omega=1, \quad \varepsilon=0.05, \quad \beta=2, \quad \gamma=2$

as in [26]. This system consists of a pendulum equation $\ddot{x}=-\sin x$, which for positive $\varepsilon$ includes a damping term $\varepsilon \beta \dot{x}$, and an harmonic oscillator $\ddot{z}=-\omega^{2} z$, which for positive $\varepsilon$ also includes a damping term $\varepsilon \gamma \dot{z}$. For $\varepsilon \neq 0$, these systems are coupled by the terms $\varepsilon(z-x)$ and $\varepsilon(x-z)$, respectively. The pendulum is also subject to a bounded perturbation $\varepsilon u(t)$, where $u$ takes values in a compact interval $U=[-\rho, \rho], \rho>0$.

The pendulum has the hyperbolic equilibria $\xi_{ \pm}$ with coordinates $\left(x_{1}, x_{2}\right)=( \pm \pi, 0)$. Together with the equilibrium $x_{3}=x_{4}=0$ of the harmonic oscillator, they constitute two non-hyperbolic equilibria of the four-dimensional system. For constant $u$ and small $\varepsilon \neq 0$, there are unique equilibria $\xi_{ \pm}^{\varepsilon}(u)$ near $\xi_{ \pm}$. They are obtained by setting

$x_{2}=x_{4}=0$,

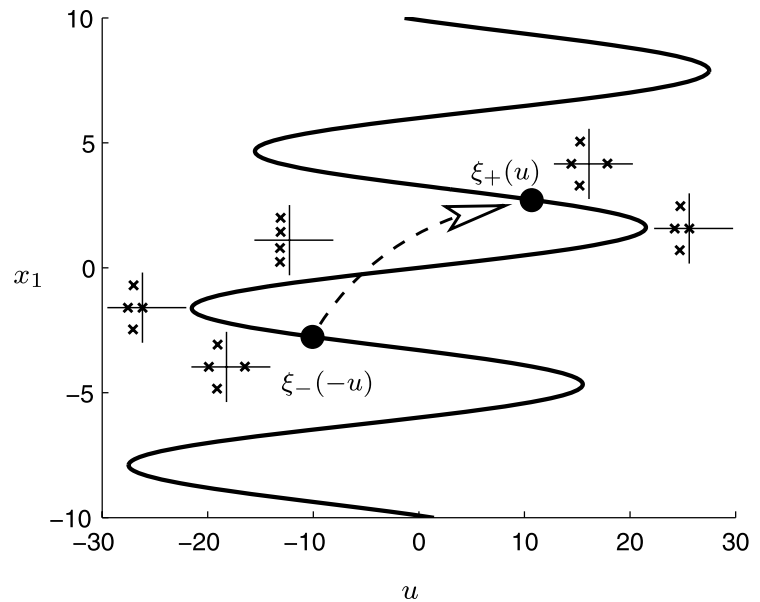

Fig. 5 Continuation of equilibria w.r.t. the control $u$, computed using the bifurcation and continuation toolbox MATCONT, cf. [10]. The arrow indicates the existence of a heteroclinic orbit of the time- $2 \pi$ map (2) with control (3), and the small diagrams show the eigenvalues of the linearization indicating the stability of these equilibria

and then from the last equation one obtains

$0=-\omega^{2} x_{3}+\varepsilon\left(x_{1}-x_{3}\right)$,

and hence

$x_{3}=\frac{\varepsilon x_{1}}{\varepsilon+\omega^{2}}$.

Thus, by the second equation, the $x_{1}$-component is the unique solution near $x_{1}=0$ of

$\sin x_{1}=\varepsilon x_{1}\left(\frac{\varepsilon}{\varepsilon+\omega^{2}}-1\right)+\varepsilon u$.

The continuation of equilibria of (9) with respect to $u$ is shown in Fig. 5.

Note the following symmetry relation: for every solution $x(t)$ in $\mathbb{R}^{4}$, one finds that $y(t):=-x(t)$ solves

$\dot{y}_{1}=y_{2}$,

$\dot{y}_{2}=-\sin y_{1}+\varepsilon\left(y_{3}-y_{1}\right)-\varepsilon \beta y_{2}-\varepsilon u(t)$,

$\dot{y}_{3}=y_{4}$,

$\dot{y}_{4}=-\omega^{2} y_{3}+\varepsilon\left(y_{1}-y_{3}\right)-\varepsilon \gamma y_{4}$.

Thus, the solutions satisfy the symmetry relation

$\varphi(t, x, u)=-\varphi(t,-x,-u)$.

Denote the time- $T$ map by $f$ and choose $T=2 \pi$. This results in a discrete-time control system of the 
form (2). We assume that for the parameter values (10) and for constant control $u \in U$, there exists an equilibrium $\xi_{+}(u)$ near $\xi_{+}$with non-trivial stable subspace and an equilibrium $\xi_{-}(-u)$ near $\xi_{-}$with nontrivial unstable subspace, such that the sum of the dimensions of these two subspaces is 4, see Fig. 5. Our goal is to compute a connecting orbit between $\xi_{-}(-u)$ and $\xi_{+}(u)$, using the algorithm, introduced in Sect. 2.

\subsection{Numerical computations}

In the numerical experiments, we chose $n_{+}=-n_{-}=$ 10 , and it turned out that the computation of heteroclinic orbits is easier for large control values. Hence, the solution of $\Gamma_{J}\left(x_{J}\right)=0$ is first computed for $u=9$, and then it was possible via parameter continuation to detect all controls for which heteroclinic orbits exist.

The first orbit, computed for $u=9$, is shown in Fig. 6; black circles denote the points of this orbit. These points are connected by the solution of (9). Dotted and dashed lines indicate the extension of the solutions for $t>0$ and $t<0$, if the control has constant value $u$ and $-u$, respectively. Note that $\min _{ \pm}\left\{\| x^{n}-\right.$ $\left.\xi_{ \pm}( \pm u) \|\right\}$ converges exponentially fast towards to 0 as $n \rightarrow \pm \infty$; see Fig. 7. Due to this rapid convergence, only the point $x^{0}$ is visible in Fig. 6, while all other points practically coincide with the fixed points $\xi_{-}(-u)$ or $\xi_{+}(u)$.

A continuation diagram of this orbit with respect to $u$ is given in Fig. 8. Starting at the initial orbit at $u=9$ (symbol (1) the algorithm of pseudo arclength continuation (see Keller [19] and Govaerts [13]) is applied in both directions $(u<9, u>9)$.

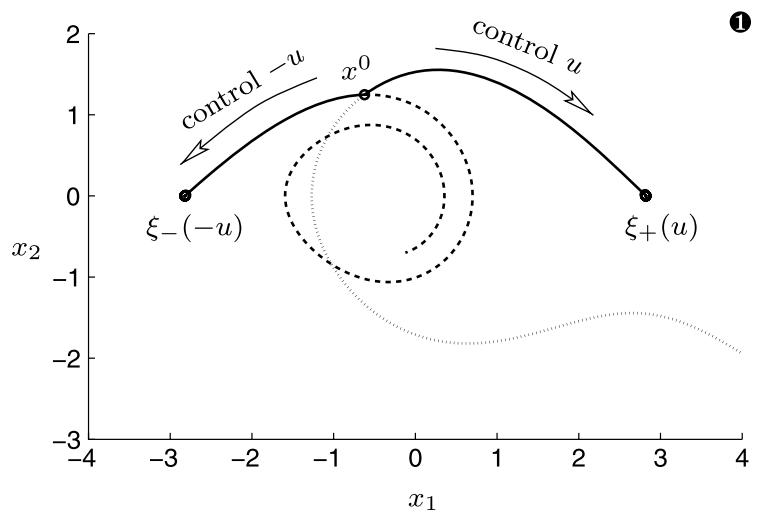

Fig. 6 Heteroclinic orbits of (2) for $u=9$

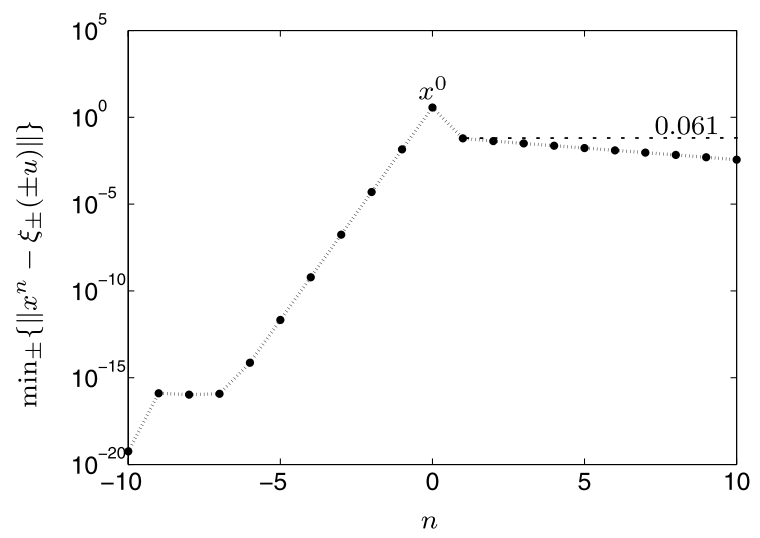

Fig. 7 Distance of the orbit from Fig. 6 to the fixed points in a logarithmic scale
Fig. 8 Continuation of heteroclinic orbits of (2) w.r.t. the control $u$. The numbers refer to the corresponding orbits from Figs. 6, 9, 10

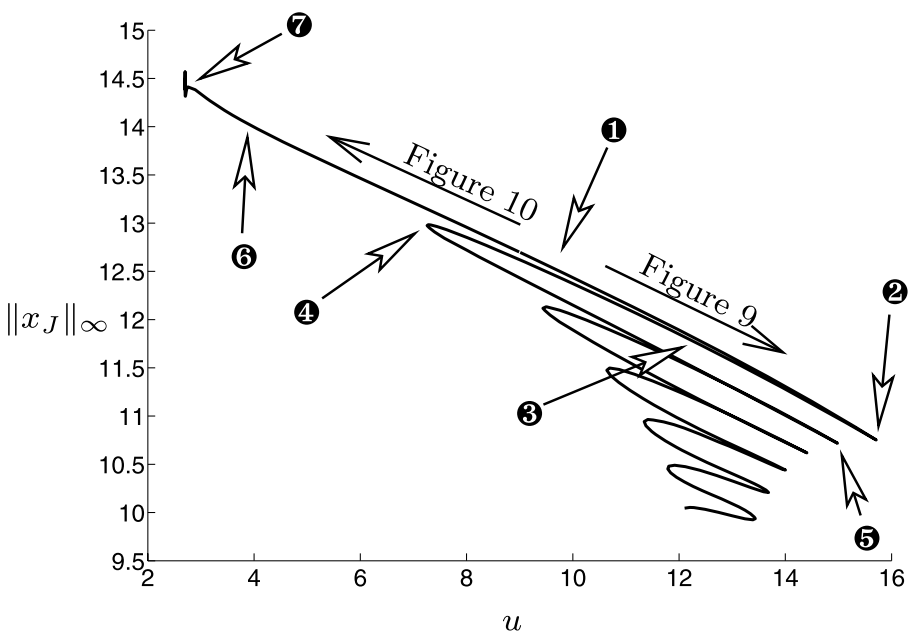


$u \approx 15.7$

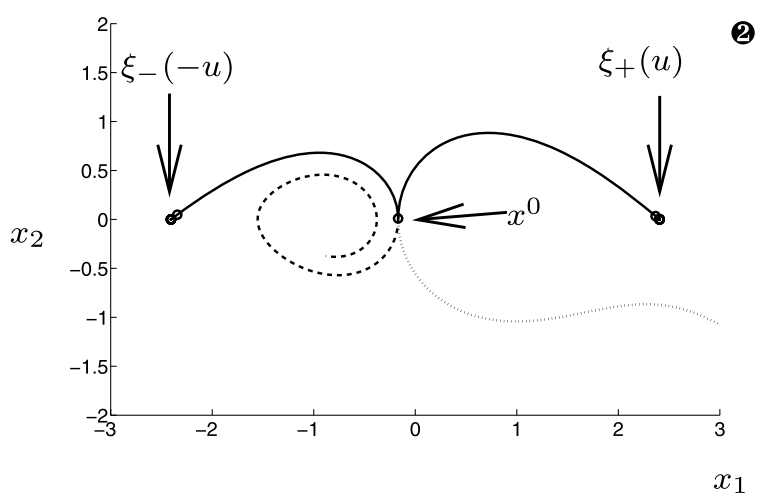

$u \approx 7.3$

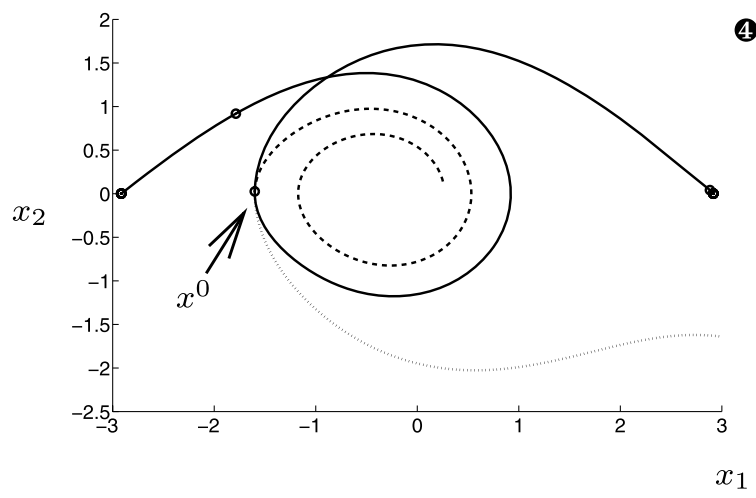

$u \approx 12$

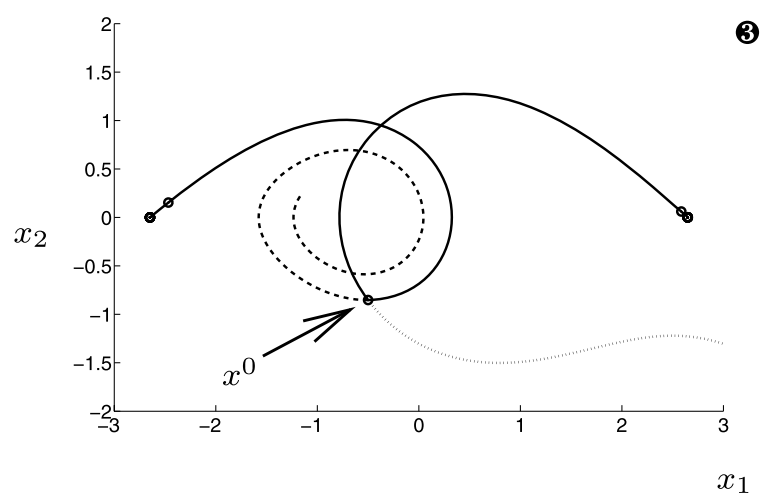

$u \approx 14.3$

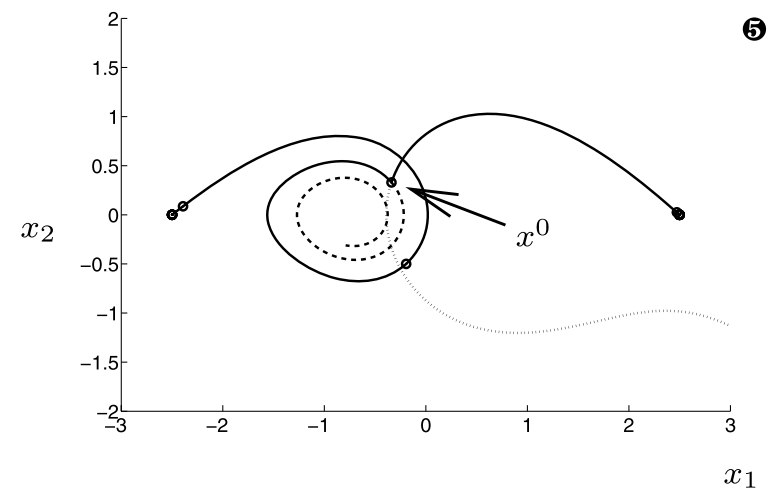

Fig. 9 A sequence of heteroclinic orbits, computed using parameter continuation; cf. Fig. 8

The continuation of orbit 1 in the direction $u>9$ exhibits a turning point at $u \approx 15.7$, denoted by 2 . There, the control parameter $u$ returns, while the center point $x^{0}$ of the orbit follows a spiraling curve; cf. orbit 2 in Fig. 9. As a consequence, the continuation of (2) in this direction exhibits an infinite number of turning points, see Fig. 9, and $x^{0}$ approaches the stable fixed point in the center of the spiral.

Characteristic orbits that occur, when continuing the initial orbit for $u<9$ are shown in Fig. 10. For $u=2.701$, there exists a heteroclinic orbit $\tilde{x}_{\mathbb{Z}}$ of the system with constant control, i.e.,

$\tilde{x}^{n+1}=f\left(\tilde{x}^{n}, \tilde{u}\right), \quad \lim _{n \rightarrow \pm \infty} \tilde{x}^{n}=\xi_{ \pm}(\tilde{u})$.

Note that this autonomous heteroclinic connection of $\xi_{-}(u)$ to $\xi_{+}(u)$ can only be found in a small neighborhood of $\tilde{u}$. Our controlled systems (2), (3) possesses for $u$ close to $\tilde{u}$ a heteroclinic connection from $\xi_{-}(-u)$ to $\xi_{+}(u)$ with an intermediate visit of the fixed point $\xi_{-}(u)$ after switching control (see Fig. 10, $\boldsymbol{\nabla}$ ). From then on, the branch stays in the $\tilde{u}$ neighborhood with more and more points accumulating near $\xi_{-}(u)$.

These computations show that heteroclinic orbits of our controlled system exist for $u \in[2.701,15.7]$.

\subsection{Controllability}

The following discussion clarifies the controllability behavior of system (9). The reachable set from $x \in \mathbb{R}^{4}$ is defined by

$\mathcal{O}^{\varepsilon,+}(x)=\left\{\varphi^{\varepsilon}(t, x, u): t \geq 0\right.$ and $\left.u \in \mathcal{U}\right\}$.

Observe that for $\varepsilon=0$, the system then does not depend on the control function, and hence, the reachable set $\mathcal{O}^{0,+}(x)$ has void interior in $\mathbb{R}^{4}$. In order to discuss 


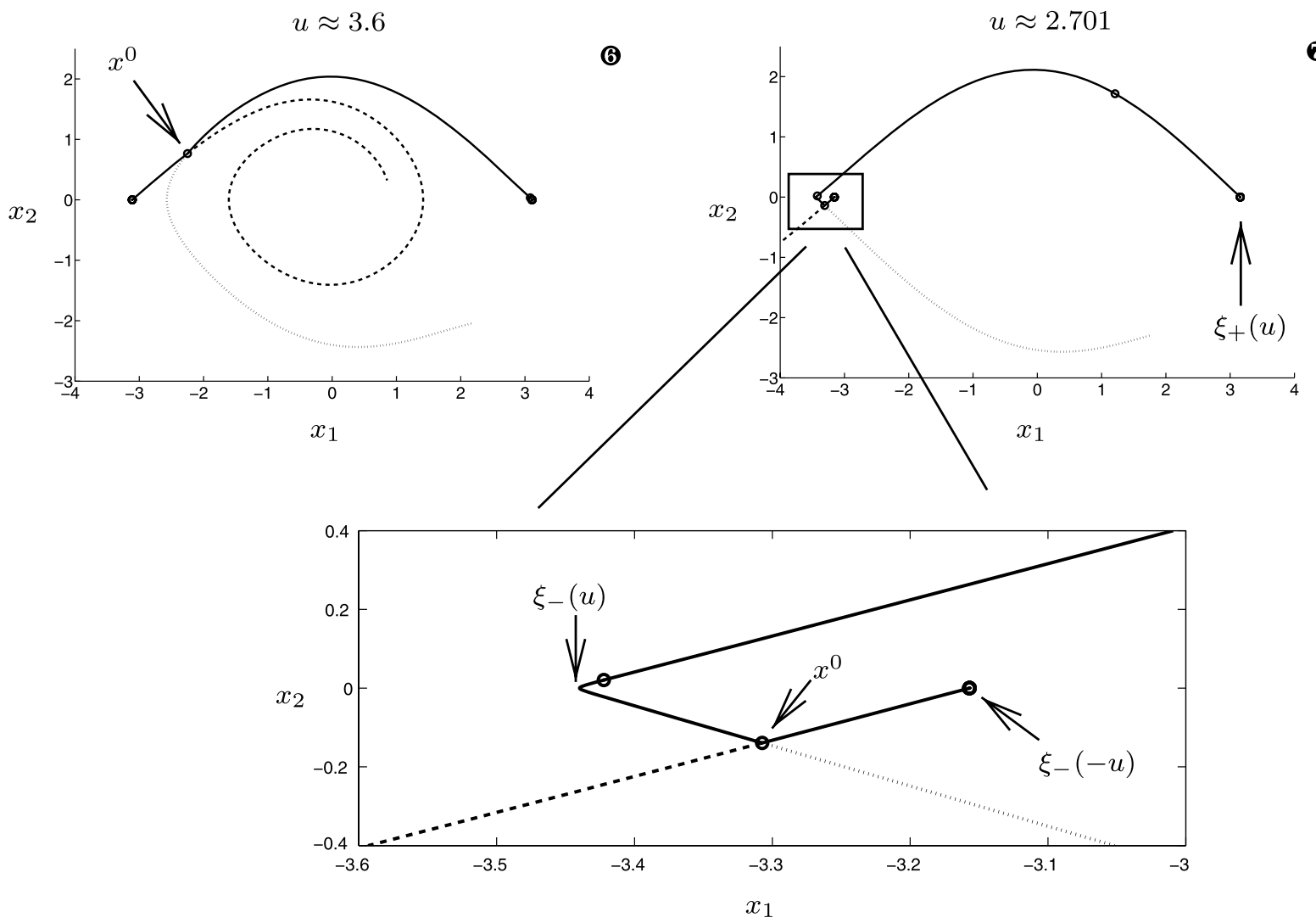

Fig. 10 A sequence of heteroclinic orbits, computed using parameter continuation; cf. Fig. 8

the controllability behavior for $\varepsilon \neq 0$, we recall the following results from Colonius and Kliemann [7]. Let $X$ and $Y$ be vector fields on $\mathbb{R}^{d}$. In the canonical local coordinates $X(x)=\alpha(x)=\left(\alpha_{1}(x), \ldots, \alpha_{d}(x)\right)^{T}$ and $Y(x)=\beta(x)=\left(\beta_{1}(x), \ldots, \beta_{d}(x)\right)^{T}$, the Lie bracket is given by

$[X, Y]=D \beta(x) \alpha(x)-D \alpha(x) \beta(x)$,

where $D \alpha(x)$ and $D \beta(x)$ denote the Jacobians. We use the notation

$\operatorname{ad}_{X}^{0} Y:=Y, \quad \operatorname{ad}_{X}^{k+1} Y:=\left[X, \operatorname{ad}^{k} Y\right]$ for all $k \geq 0$.

Theorem 1 Consider a control-affine system of the form

$\dot{x}=X(x)+u(t) Y(x), \quad u(t) \in U$,

where $X$ and $Y$ are smooth vector fields on $\mathbb{R}^{d}$ and $U$ is an interval containing the origin in its interior.
Suppose that

$\left.\operatorname{span}\left\{\operatorname{ad}_{X}^{k} Y\right)(x): k \in \mathbb{N}_{0}\right\}=\mathbb{R}^{d}$ for all $x \in \mathbb{R}^{d}$.

Then every equilibrium of the uncontrolled system $\dot{x}=$ $X(x)$ is an interior point of a control set D, i.e., a maximal subset $D$ of $\mathbb{R}^{d}$ such that $D \subset \mathrm{clO}^{+}(x)$ for all $x \in D$. Furthermore, one has int $D \subset \mathcal{O}^{+}(x)$ for all $x \in D$.

Proof Hypothesis (15) implies that the system without control constraints is strongly locally accessible (cf. Nijmeier and van der Schaft [22, Theorem 3.21]). Hence, by [7, Proposition 4.5.17], the system with control constraints satisfies the so-called inner-pair condition guaranteeing by [7, Corollary 4.5.11] that every equilibrium of the uncontrolled system is contained in the interior of a control set. The second assertion follows from [7, Lemma 3.2.13(ii)], since the inner-pair condition implies local accessibility. 
System (9) is a special case of (14) with $d=4$ and vector fields

$X(x)=\left(\begin{array}{c}x_{2} \\ -\sin x_{1}+\varepsilon\left(x_{3}-x_{1}\right)-\varepsilon \beta x_{2} \\ x_{4} \\ -\omega^{2} x_{3}+\varepsilon\left(x_{1}-x_{3}\right)-\varepsilon \gamma x_{4}\end{array}\right)$

and

$Y(x)=\left(\begin{array}{c}0 \\ -\varepsilon \\ 0 \\ 0\end{array}\right)$.

Since the vector field $Y$ is constant, its Jacobian vanishes, and the Jacobian of $X$ is given by

$$
\left(\begin{array}{cccc}
0 & 1 & 0 & 0 \\
-\cos x_{1}-\varepsilon & -\varepsilon \beta & \varepsilon & 0 \\
0 & 0 & 0 & 1 \\
\varepsilon & 0 & -\omega^{2}-\varepsilon & -\varepsilon \gamma
\end{array}\right) .
$$

One computes the Lie bracket as

$$
\begin{aligned}
\operatorname{ad}_{X}^{1} Y= & \left(\begin{array}{cccc}
0 & 1 & 0 & 0 \\
-\cos x_{1}-\varepsilon & -\varepsilon \beta & \varepsilon & 0 \\
0 & 0 & 0 & 1 \\
\varepsilon & 0 & -\omega^{2}-\varepsilon & -\varepsilon \gamma
\end{array}\right) \\
& \times\left(\begin{array}{l}
0 \\
\varepsilon \\
0 \\
0
\end{array}\right)=\varepsilon\left(\begin{array}{c}
1 \\
-\varepsilon \beta \\
0 \\
0
\end{array}\right) .
\end{aligned}
$$

Similarly, one computes

$\operatorname{ad}_{X}^{2} Y=-\varepsilon\left(\begin{array}{c}-\varepsilon \beta \\ -\cos x_{1}-\varepsilon+\varepsilon^{2} \beta^{2} \\ 0 \\ \varepsilon\end{array}\right)$

and

$\operatorname{ad}_{X}^{3} Y=\varepsilon\left(\begin{array}{c}\cos x_{1}+\varepsilon-\varepsilon^{2} \beta^{2} \\ x_{2} \sin x_{1}-\varepsilon \beta\left(2 \cos x_{1}+2 \varepsilon-\varepsilon^{2} \beta^{2}\right) \\ -\varepsilon \\ \varepsilon^{2}(\beta+\gamma)\end{array}\right)$.

For $\varepsilon \neq 0$, the four vector fields $Y, \operatorname{ad}_{X}^{1} Y, \operatorname{ad}_{X}^{2} Y, \operatorname{ad}_{X}^{3} Y$ are linearly independent for all points $x \in \mathbb{R}^{4}$, since

$\operatorname{det}\left(Y, \operatorname{ad}_{X}^{1} Y, \operatorname{ad}_{X}^{2} Y, \operatorname{ad}_{X}^{3} Y\right)=\varepsilon^{3}$.
This shows that the assumptions of Theorem 1 are satisfied. It follows that the equilibria $\xi_{+}^{\varepsilon}(0)$ and $\xi_{-}^{\varepsilon}(0)$ of the uncontrolled system are contained in the interiors of control sets, which we denote by $D_{+}^{\varepsilon}$ and $D_{-}^{\varepsilon}$, respectively. The equilibria $\xi_{+}^{\varepsilon}(u)$ and $\xi_{-}^{\varepsilon}(u)$ given by (11) and (12) depend continuously on $u$ and $\varepsilon$. By applying Theorem 1 again, one finds that, for $u \in \operatorname{int} U$, they are contained in the interiors of $D_{+}^{\varepsilon}$ and $D_{-}^{\varepsilon}$, respectively. The constructed heteroclinic orbit approaches for $t \rightarrow \infty$ the equilibrium $\xi_{+}^{\varepsilon}(u) \in \operatorname{int} D_{+}^{\varepsilon}$ and for $t \rightarrow-\infty$ the equilibrium $\xi_{-}^{\varepsilon}(-u) \in \operatorname{int} D_{-}^{\varepsilon}$ connecting the control sets $D_{+}^{\varepsilon}$ and $D_{-}^{\varepsilon}$ by a finite time controlled trajectory. By local accessibility and the second part of Theorem 1, one can connect any two points in the interior of the $D_{+}^{\varepsilon}$; analogously in $D_{-}^{\varepsilon}$. Hence our result shows that the equilibria $\xi_{-}^{\varepsilon}(-u)$ and $\xi_{+}^{\varepsilon}(-u)$ are, in fact, connected by a controlled trajectory in finite time. However, it seems rather difficult to determine it directly instead of following our approach above. Taking into account the symmetry property (13) one sees that there is also a trajectory from $D_{+}^{\varepsilon}$ to $D_{-}^{\varepsilon}$. Since control sets are maximal sets of complete controllability, these two control sets coincide. We conclude that there is a single control set containing all equilibria as well as the heteroclinic connections.

\subsection{Random perturbations}

Finally, we briefly discuss consequences of our results in the case of random perturbations. Consider, instead of deterministic functions $u(t)$, random perturbations of the form

$\zeta(t)=\rho \sin (\Omega t+\sigma w(t))$,

where $\rho$ and $\Omega$ are the amplitude and averaged frequency of bounded noise and $w(t)$ is the Wiener process with intensity $\sigma$ (essentially, this is the situation considered in Zhu and Liu [26, Sect. 2]). Then $\eta(t):=\Omega t+\sigma w(t)$ may be considered on the unit circle, a compact manifold parameterized by $[0,2 \pi)$. Now it follows (compare Colonius and Kliemann [6]) that for every deterministic control function $u(t)$ taking values in the interval $U=[-\rho, \rho]$ and every $T>0$ every tube around a trajectory $\varphi(t, x, u)$, $t \in[0, T]$, has positive probability. Our numerical results show that there is a control function $u_{1}$ with values in the interval [-2.702, 2.702], such that the corresponding trajectories start in a neighborhood of $\xi_{-}$, visit a neighborhood of $\xi_{+}$, and then return into the 
neighborhood of $\xi_{-}$. It also follows from the discussion above that there is a control function $u_{2}$ such that the corresponding trajectory moves around two times, and similarly for arbitrary $n \in \mathbb{N}$. Consequently, for every $n \in \mathbb{N}$, the random system has positive probability that there are trajectories moving around $n$ times.

\section{Conclusion}

A Newton method for computation of connecting orbits in non-linear systems with deterministic or random perturbations is presented. A major advantage of this approach, compared to methods based on intersections of stable and unstable manifolds, is that it can be used for higher-dimensional systems. The efficiency of the method is demonstrated for a two-dimensional model of ship roll motion with sinusoidal forcing and for a four-dimensional system consisting of a pendulum coupled with a harmonic oscillator. Combining this analysis with methods from control theory and random dynamics, one finds consequences for controllability behavior and for random behavior. It should be noted that these methods only give the information, if probabilities are positive, not their actual magnitude.

Acknowledgement The authors wish to thank the two anonymous referees for valuable suggestions, leading to an improvement of this paper.

\section{References}

1. Beyn, W.-J.: The numerical computation of connecting orbits in dynamical systems. IMA J. Numer. Anal. 10(3), 379-405 (1990)

2. Beyn, W.-J., Hüls, T.: Error estimates for approximating non-hyperbolic heteroclinic orbits of maps. Numer. Math. 99(2), 289-323 (2004)

3. Beyn, W.-J., Hüls, T., Kleinkauf, J.-M., Zou, Y.: Numerical analysis of degenerate connecting orbits for maps. Int. J. Bifurc. Chaos 14(10), 3385-3407 (2004)

4. Beyn, W.-J., Kleinkauf, J.-M.: The numerical computation of homoclinic orbits for maps. SIAM J. Numer. Anal. 34(3), 1207-1236 (1997)

5. Colonius, F., Kapela, T.: A rigorous numerical algorithm for controllability. In: Lamnabhi-Lagarrigue, F., Laghrouche, S., Loria, A., Panteley, E. (eds.) Taming Heterogeneity and Complexity of Embedded Control. CTSHYCON Workshop on Nonlinear and Hybrid Control. International Scientific \& Technical Encyclopedia (ISTE), pp. 157-170. London (2007)

6. Colonius, F., Kliemann, W.: Topological, smooth, and control techniques for perturbed systems. In: Crauel, H.,
Gundlach, M. (eds.) Stochastic Dynamics, pp. 181-208. Springer, New York (1999)

7. Colonius, F., Kliemann, W.: The Dynamics of Control. Birkhäuser, Boston (2000)

8. Colonius, F., Marquardt, A., Kreuzer, E., Sichermann, W.: A numerical study of capsizing: comparing control set analysis and Melnikov's method. Int. J. Bifurc. Chaos 18(5), 1503-1514 (2008)

9. Demmel, J.W., Dieci, L., Friedman, M.J.: Computing connecting orbits via an improved algorithm for continuing invariant subspaces. SIAM J. Sci. Comput. 22(1), 81-94 (2000)

10. Dhooge, A., Govaerts, W., Kuznetsov, Y.A.: MATCONT: a MATLAB package for numerical bifurcation analysis of ODEs. ACM Trans. Math. Softw. 29(2), 141-164 (2003)

11. Frey, M., Simiu, E.: Noise-induced chaos and phase space flux. Physica D, Nonlinear Phenom. 63(3-4), 321-340 (1993)

12. Gayer, T.: Control sets and their boundaries under parameter variation. J. Differ. Equ. 201(1), 177-200 (2004)

13. Govaerts, W.J.F.: Numerical Methods for Bifurcations of Dynamical Equilibria. SIAM, Philadelphia (2000)

14. Guckenheimer, J., Holmes, P.: Nonlinear Oscillation, Dynamical Systems, and Bifurcations of Vector Fields. Applied Mathematical Sciences, vol. 42. Springer, New York (1983)

15. Häckl, G., Schneider, K.R.: Controllability near TakensBogdanov points. J. Dyn. Control Syst. 2(4), 583-598 (1996)

16. Hüls, T.: Homoclinic orbits of non-autonomous maps and their approximation. J. Differ. Equ. Appl. 12(11), 11031126 (2006)

17. Hüls, T.: Homoclinic trajectories of non-autonomous maps. J. Differ. Equ. Appl. (2009, to appear)

18. Jaulin, L., Kieffer, M., Didrit, O., Walter, É.: Applied Interval Analysis. Springer, London (2001)

19. Keller, H.B.: Numerical solution of bifurcation and nonlinear eigenvalue problems. In: Applications of Bifurcation Theory, pp. 359-384. Academic Press, New York (1977)

20. Kreuzer, E., Sichermann, W.: Investigation of large amplitude roll motions and capsizing. In: Keil, H., Lehmann, E. (eds.) Proceedings of the Ninth International Symposium on Practical Design of Ships and Other Floating Structures, pp. 689-696. Hansa, Ahrensburg (2004)

21. Melnikov, V.K.: On the stability of a center for timeperiodic perturbations. Trans. Mosc. Math. Soc. 12, 3-52 (1963) (in Russian)

22. Nijmeijer, H., van der Schaft, A.: Nonlinear Dynamical Control Systems. Springer, Berlin (1990)

23. Simiu, E.: Chaotic Transitions in Deterministic and Stochastic Dynamical Systems. Princeton Series in Applied Mathematics. Princeton University Press, Princeton (2002)

24. Wichtrey, T.: Melnikov method and heteroclinic control sets. Bachelor thesis, Universität Augsburg (2006) (in German)

25. Wiggins, S.: Global Bifurcations and Chaos. Applied Mathematical Sciences, vol. 73. Springer, New York (1988)

26. Zhu, W.Q., Liu, Z.H.: Homoclinic bifurcation and chaos in coupled simple pendulum and harmonic oscillator under bounded noise excitation. Int. J. Bifurc. Chaos 15(1), 233-243 (2008) 